\title{
Voll Fett - Öle, Fette und Wachse im Pflanzenreich
}

\section{Hilke Steinecke \& Peter Schubert}

\section{Einfuihrung}

Unter dem genannten Motto standen von Januar bis April 2008 die abendlichen Führungen durch das Tropicarium. Wie in jeder Saison trafen sich zu den Veranstaltungen jeweils etwa 40 Teilnehmerinnen und Teilnehmer, um anschließend, aufgeteilt in zwei Gruppen, öl-, fett- und wachshaltige Pflanzen in den Gewächshäusern zu erkunden.

Fette und fette Öle sind Ester des dreiwertigen Alkohols Glycerin mit drei Fettsäuren. Je nachdem, ob die Substanzen bei Raumtemperatur fest oder flüssig sind, spricht man von Fetten oder fetten Ölen. Viele von ihnen werden technisch (z. B. als Schmieröle) oder als Speisefette bzw. -öle verwendet. Fette haben im Körper verschiedene Bedeutungen: als Energiespeicher, Schutz vor mechanischen Verletzungen oder Wärmeisolation. Lipide, zu denen u.a. auch Fette gehören, bauen das Membransystem der Zellen auf.

Zum Einstieg wurde eine Auswahl verschiedener Ölsaaten vorgestellt, d.h. Pflanzen, aus deren Samen oder Früchten Öl gewonnen wird. Einige Beispiele sind Sonnenblume, Ölbaum, Mango, Baumwolle, Erdnuss und Mandel. Interessanterweise haben aber auch Pflanzen, die nicht besonders viel Fett enthalten, einen „fettigen" Namen wie z. B. Speckbaum oder Fettkraut. Hinter letzterem verbergen sich fleischfressende Pflanzen der Gattung Pinguicula mit saftig-fleischigen Blättern. Die lateinische Vokabel pinguis bedeutet fett, so wie auch der Pinguin wörtlich genommen ein fetter Vogel ist.

In kleinen Experimenten wurden die Eigenschaften von Öl demonstriert, z. B. die Nichtmischbarkeit mit Wasser. An einen Vulkan erinnernd stieg rot gefärbtes Olivenöl aus einem in eine Wasserschale gestellten Röhrchen auf, um sich dann später an der Wasseroberfläche zu verteilen. Fette Öle hinterlassen Flecken auf Papier, ätherische Öle dagegen nicht. Letztere haben meist einen starken Duft und sind leicht flüchtig. Wachse können unterschiedlicher che- mischer Natur sein. Wie Fette sind auch sie wasserabweisend. Bei $20^{\circ} \mathrm{C}$ sind sie knetbar und bei etwa $40^{\circ} \mathrm{C}$ schmelzen sie.

\section{Wachse}

Im Halbwüsten-Haus gedeihen viele Kakteen, Agaven und andere Sukkulenten. Ihre Blätter bzw. Stämme sind häufig von graublau schimmernden Wachsen überzogen. Sie schützen die Pflanzen vor Strahlung und zu großen Wasserverlusten. Besonders beeindruckten hier die großen und festen bläulichen Blätter der mexikanischen Palme Brahea armata. Ähnlich wie die in Brasilien heimische Wachs- oder Carnauba-Palme (Copernicia prunifera, benannt nach Nikolaus Kopernikus) tragen die großen Fächerblätter dicke Wachsschichten. CarnaubaWachs findet viele Anwendungen und ist z. B. in Autopolituren oder Lippenstiften enthalten. Aber auch Gummibärchen sind mit diesem Wachs, oft in Kombination mit Bienenwachs, überzogen, um Wasserverlust oder ein Verkleben zu verhindern. Vermutlich kaum jemand hat vor der Führung an einen Zusammenhang zwischen einer Wolfsmilch und Körperbehaarung gedacht. Die in Trockengebieten Mexikos verbreitete Candelilla-Wolfsmilch (Euphorbia cerifera) hat stark wachsige Triebe. Auf das Wachs bezieht sich der spanische Volksname, der ,kleine Kerze" bedeutet. Das Wachs kann durch Kochen der Spross-Stücke in Wasser und anschließendes Abschöpfen gewonnen werden. In gereinigter, gleichmäßig zerkleinerter Form findet es Einsatz als Bindemittel bei der Herstellung von Künstlerfarben oder aber ist Bestandteil von Enthaarungsmitteln. Ebenfalls eine "Wachsblume" ist der Jojobastrauch (Simmondsia chinensis). Der wissenschaftliche Name ist irreführend, denn das mit dem Buchsbaum verwandte Gehölz kommt nicht in China, sondern in Arizona, Kalifornien und Mexiko vor. Das bekannte Jojobaöl aus den Samen ist kein Öl, sondern ein flüssiges Wachs. Es spielt vor allem bei der Herstellung von Kosmetika eine große Rolle. 
Da es nicht wie Fett ranzig wird, eignet es sich sehr gut als Bestandteil von Cremes, um diese geschmeidig zu machen. Außerdem schützt es die Haut vor UV-Strahlung und wird deshalb Sonnencremes zugegeben. Zur Erinnerung an diese Station gab es für jeden einen Bogen handgefertigten „Wachsblumen-Briefpapiers“, der mit einer Blüte bedruckt und mit Wachstropfen verziert war.

\section{3. Öle}

Im Savannenhaus wurde exemplarisch für die vielen ölhaltigen „Nüsse“ der MacadamianussBaum (Macadamia integrifolia) demonstriert, der im Winter gerade blühte und die ersten jungen Früchte zeigte. Reife Früchte sind von einer harten Schale umgeben, so dass sie mit Hilfe eines Spezialbohrers, der an einen Schraubstock erinnert, geöffnet werden. Wird der Bohrer in die Macadamianuss hineingedreht, tropft mitunter etwas Öl heraus. Kalorienbewusste Menschen essen vermutlich nur wenige der sehr energiereichen Macadamianüsse. Anderseits enthalten sie vor allem ungesättigte Fettsäuren und Vitamine, so dass sie auch "gesund" sind. Öle aus verschiedenen Nüssen werden zur Körperpflege eingesetzt, darunter auch das Paranuss-Öl. Die Samen des Paranuss-Baumes enthalten so viel Öl, dass sie angezündet werden können und eine Zeit lang wie eine Kerze brennen, was die Zuschauer stets sehr staunen ließ. An anderer Stelle wurden ergänzend hierzu Kokos- und Ölpalme sowie die entsprechenden Öle und Fette vorgestellt.

Gleich neben der Nuss-Station wurden Speiseöle vorgestellt. Qualität, Geschmack und Verwendung verschiedener Öle wurden erläutert. Eines der teuersten Speiseöle ist das Arganöl aus den Samen des Arganbaumes (Argania spinosa). Dieser ist in einem kleinen Gebiet in Marokko heimisch. Traditionell gewinnen Berberfrauen das Öl in langwieriger Handarbeit aus den extrem harten Samen, deren Schale etwa $16 \mathrm{Mal}$

Abb. 1 (oben): Der Name Fettkraut für diese fleischfressende Pflanze bezieht sich auf ihre fleischigen Blätter.

Abb. 2 (unten): Brennende Paranuss.
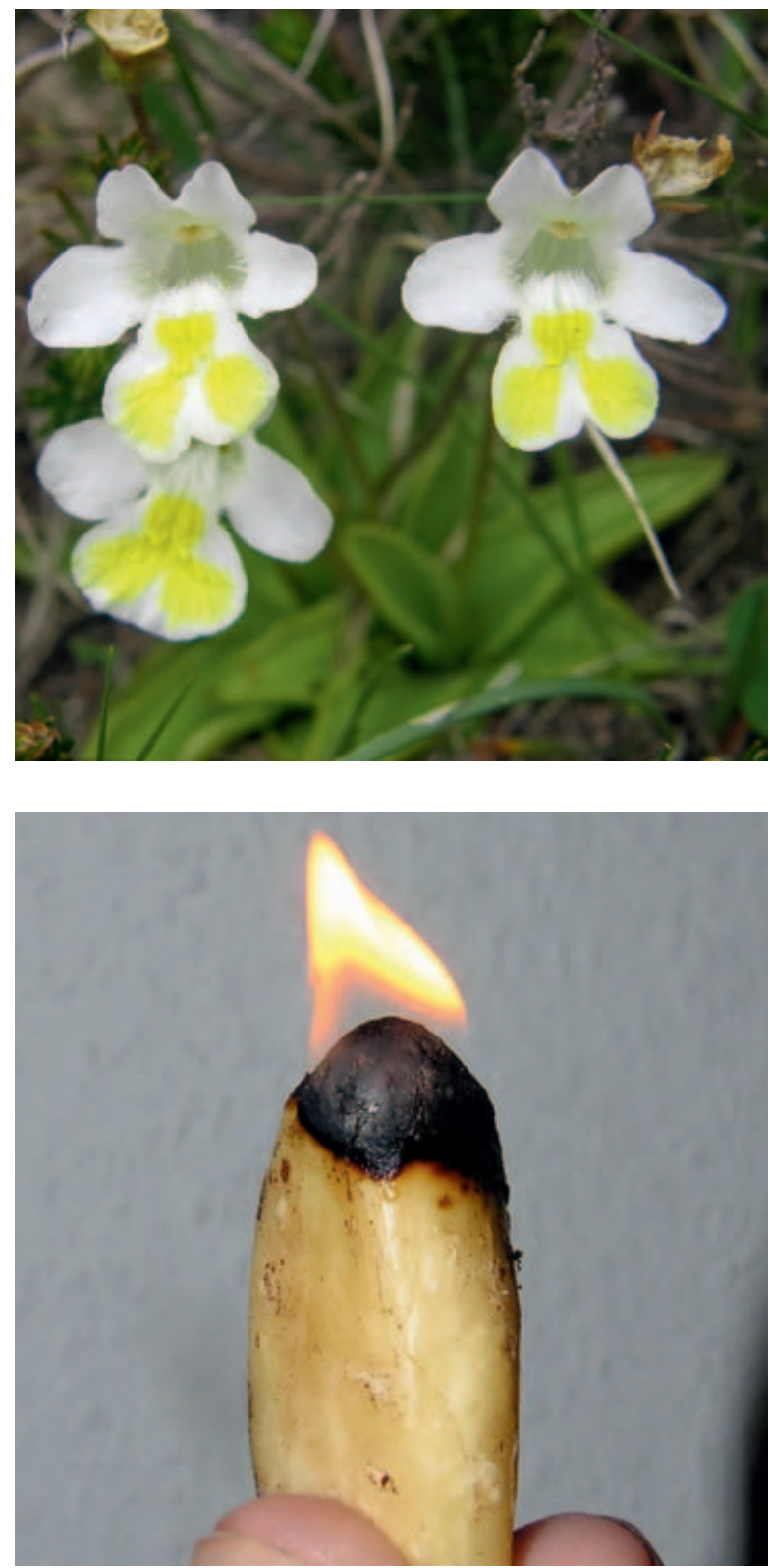

so hart wie diejenige einer Haselnuss sein soll. Bei uns bekommt man Arganöl zu kaufen, das entweder aus gerösteten oder ungerösteten Samen gewonnen wurde. Die Teilnehmenden konnten sich von dem ganz unterschiedlichen Geruch beider Sorten überzeugen.

Gerade nach Weihnachten möchte manch einer sein Fett weg bekommen. Es gibt viele Vorschläge für eine Diät. In der Nebelwüste zeigten wir Hoodia gordonii. Es handelt sich um einen Vertreter der Seidenblumengewächse aus der Kalahari-Wüste. Buschmänner essen seit Jahrhunderten Hoodia als Appetitzügler. Die appetit- und dursthemmende Wirkung wurde in den 1960er Jahren bekannter, als Fährten- 


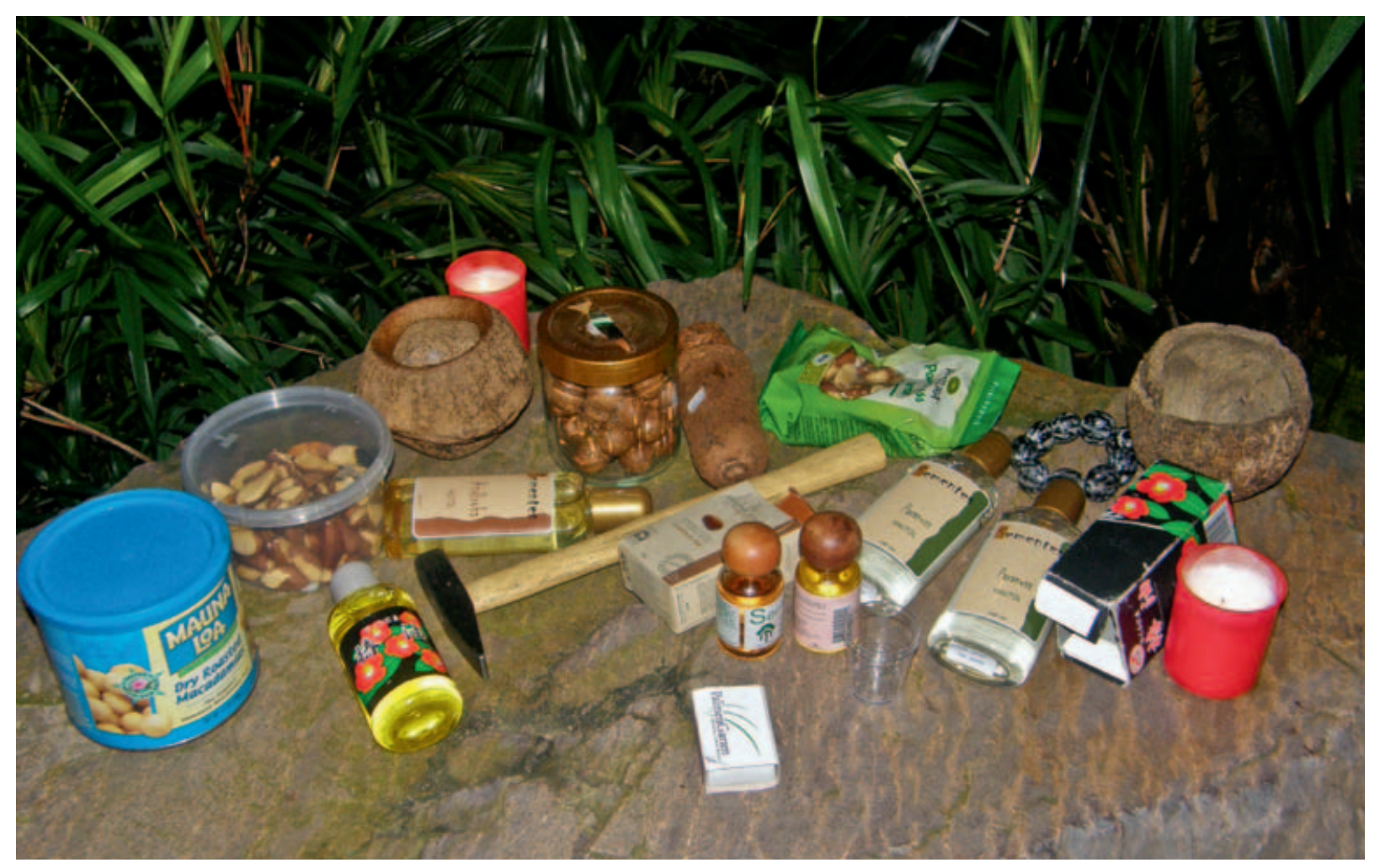

leser im Angolakrieg kaum Nahrung aufnahmen und nur gelegentlich Hoodia-Stücke aßen. Über diese Wirkung gibt es jedoch noch keine ausreichenden wissenschaftlichen Erkenntnisse. Mittlerweile sind zahlreiche Hoodia-Präparate auf dem Markt. Sie enthalten Hoodia-Pulver (aus getrockneten Sprossen), aber niemals einen Extrakt. Hoodia soll auch als Heilmittel gegen Husten und Erkältungskrankheiten sowie als ein Aphrodisiakum wirksam sein.

\section{4. Ätherische Öle}

Im Monsun- und Regenwaldhaus gedeihen zahlreiche Gewürzpflanzen, ein Anlass, hier ätherische Öle vorzustellen. Gewürze enthalten in Rinde, Blättern, Blüten, Früchten oder Samen meist einen großen Anteil an ätherischen Ölen. Es wurde z. B. an einer dicken Zimtstange geschnuppert und jeder bekam eine Zimtblüte zum Probieren. Zimtblüten sind keine Blüten, sondern die fast reifen Früchte des Zimtbaumes. Sie können wie die Rinde zum Würzen verwendet werden und erinnern in ihrer Form etwas an Gewürznelken. Während des Zerkauens verändert sich ihr Geschmack von intensiv zimtartig nach süßlich. Mit Hilfe eines mit Wasser gefüllten Glases wurde anschließend probiert, ob
Gewürznelken noch frisch und aromatisch sind. Enthalten sie in ihren Köpfen reichlich ätherisches Öl, sind sie also noch stark aromatisch, richten sich die Nelken im Wasser auf, da die leichten Köpfe sich an der Wasseroberfläche sammeln. Werden frische Nelken zerdrückt, tritt ätherisches Öl aus. Als weitere wichtige aromatische Pflanze wurde Melaleuca alternifolia vorgestellt. Aus den Blättern des Gehölzes wird das als Allheilmittel verwendete Teebaumöl gewonnen. Die Pflanze hat nichts mit der Teepflanze (Camellia sinensis) zu tun und ist mit Myrthe und Eukalyptus verwandt. Sie erhielt ihren Volksnamen von JaMes CoOK, der sich aus den Blättern einen aromatischen Tee aufbrühte.

\section{Körperbutter}

$\mathrm{Zu}$ Beginn der Führung hatten wir versprochen, dass jeder sein Fett wegbekommt, hoffentlich aber niemand in ein Fettnäpfchen tritt. Im Bromeliarium war es soweit. Neben dem Kakaobaum, der an seinem Stamm dicke gelbe Früchte

Abb. 3: Station fette „Nüsse“. 
trug, gab es Erläuterungen zur Kakaobutter. Es gab Kakaobutter, die außer in Süßigkeiten auch in vielen Cremes vorhanden ist, zu kosten. Ihr Geschmack erinnert an Weiße Schokolade. Da sie bei Körpertemperatur schmilzt, wurde sie früher auch zum Ummanteln von Zäpfchen benutzt.

Sheabutter aus den Samen des westafrikanischen Sheabutterbaumes (Karitébaum, Vitellaria paradoxa) findet in der Kosmetikindustrie immer mehr Verwendung. Die Beerenfrüchte des Baumes enthalten ca. $4 \mathrm{~cm}$ große Samen, die sog. Kariténüsse. Diese bestehen zu etwa $50 \%$ aus Fett und stellen eine wesentliche Quelle für Speise- und Hautpflegefett dar. Das Fruchtfleisch ist essbar. Aus Sheabutter und oder Kakaobutter kann man sich zu Hause leicht selber einen Körperbalsam herstellen. Dazu wird das Fett im Wasserbad langsam geschmolzen. Wenn es flüssig ist, gibt man etwa 20 Vol\% Jojobaöl dazu, verrührt alles gut und lässt die Masse abkühlen und fest werden. Dazu können einige Tropfen ätherisches Öl für einen guten Duft des Balsams gegeben werden. Ein Klümpchen „Palmengarten-Balsam“, verpackt in Zellophanpapier, konnte jeder mitnehmen.

In vielen Körperpflegemitteln ist auch Mangobutter enthalten. Mango (Mangifera indica) ist ein Vertreter der Anacardiaceae. In dieser Pflanzenfamilie gibt es viele fetthaltige Vertreter, darunter z. B. der Cashewbaum (Anacardium occidentale). Für den Freund besonderer Liköre ist aus dieser Familie vor allem der Marulabaum (Elefantenbaum, Sclerocarya birrea) interessant. Die aprikosenähnlichen Früchte enthalten einen fetthaltigen Samen und ein saftig-süßes Fruchtfleisch. Die Früchte werden gern von Elefanten gefressen (daher der deutsche Name). Sie dienen aber auch zur Herstellung des Amarula-Likörs. Da die frischen Früchte schnell verderben, werden sie selten exportiert.

Abb. 4 (oben): Fruchtender Kakaobaum; aus den Samen wird Kakaobutter gewonnen.

Abb. 5 (unten): Das Nährgewebe in den Kokosnüssen ist sehr fetthaltig.
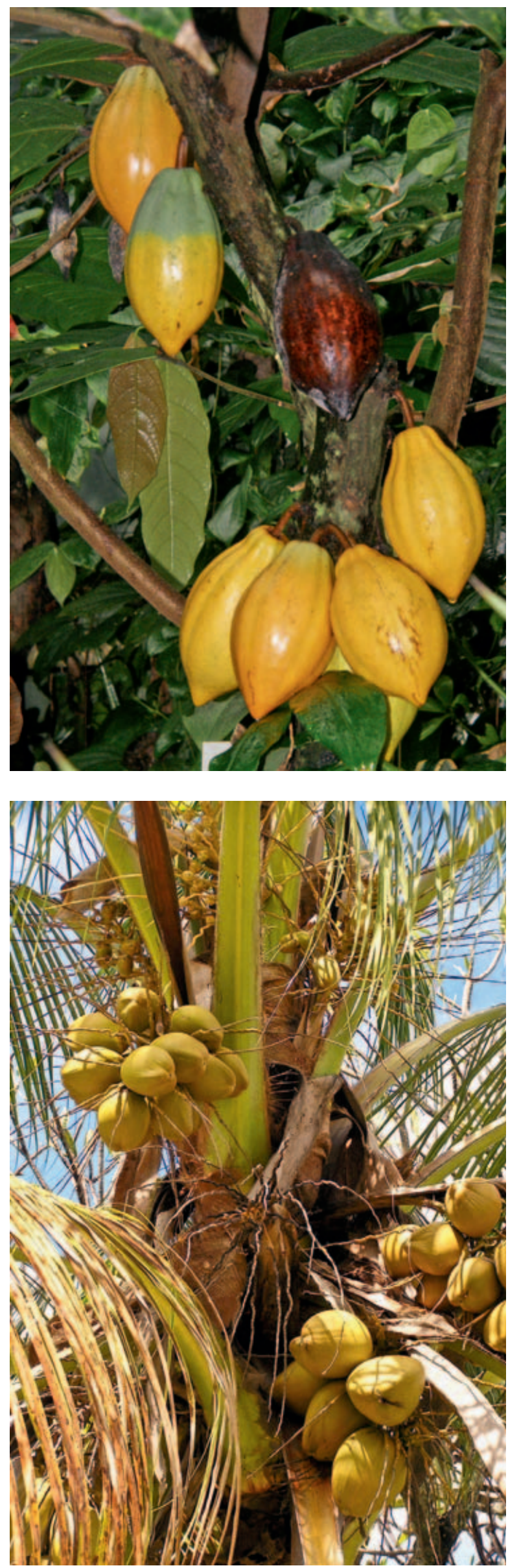


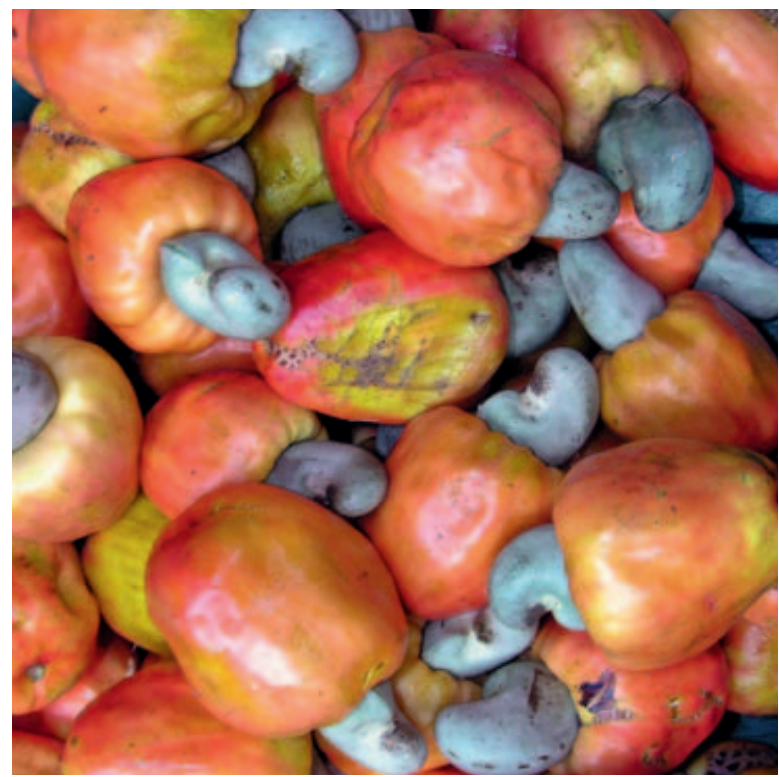

\section{6. Öle als Insektizide}

Bei einem spätabendlichen Spaziergang durch das Tropicarium fallen immer wieder die vielen dicken Schaben auf. Auch andere Schadinsekten machen den Pflanzen zu schaffen. Im Palmengarten wird auf den Einsatz von Gift weitgehend verzichtet, die Schädlinge werden durch Nützlinge oder für den Menschen ungiftige Mittel kontrolliert.

Ein sehr effektives Pflanzenschutzmittel ist das Neemöl aus den Samen des indischen Neembaumes (Azadirachta indica). Der ursprünglich aus Indien stammende Neembaum ist ein Lebenskünstler, der Temperaturen bis $50^{\circ} \mathrm{C}$ erträgt. Er eignet sich daher zum Anbau in kargen Gebieten und dient dort als Schattenspender. Der Neembaum ist immergrün und wird $30 \mathrm{~m}$ hoch und bis 100 Jahre alt. Er gilt als „Dorfapotheke“, denn er fördert die Gesundheit von Pflanze, Mensch und Tier. In Indien ist Neemöl Bestandteil des täglichen Lebens, da es gegen Krankheiten und Verletzungen genutzt wird, aber auch gegen Parasiten hilft oder in der Kosmetik (Shampoo, Seife) Verwendung findet. Bei uns wird der Neembaum vor allem als biologisches Pflanzenschutzmittel genutzt. Aus den etwa olivenkerngroßen Samen wird das Neemöl gepresst. Es enthält als besonderen Wirkstoff das Azadirachtin. Es vertreibt diverse Schädlinge bzw. hemmt die Produktion von Häutungshormonen und somit die Entwick-
lungderTiere. Neemschrotaus denzerkleinerten Samenschalen kann dem Pflanzenkultursubstrat als Dünger zugegeben werden. Im Handel gibt es zudem Lavendelsäckchen mit Neemsamen zum Vertreiben von Motten aus dem Kleiderschrank.

\section{Sporen}

Gegen Ende der Führung wurde eindrucksvoll demonstriert, warum bestimmte Oberflächen pflanzlicher Strukturen fetthaltig bzw. wasserabweisend sein sollten. Sporen von Farnen und Bärlappen werden meist mit Hilfe des Windes durch die Luft ausgebreitet. Damit sie bei hoher Luftfeuchtigkeit nicht verklumpen und damit zu schwer werden, ist die äußere Sporenwand wasserabstoßend. Wir führten den Teilnehmenden Lycopodium-Sporen vor. Die Bärlappsporen wurden in ein Wasserglas geschüttet. Sie bilden auf der Wasseroberfläche eine trockene, staubige Schicht. Steckt man den Finger in das Wasser, schmiegt sich das Sporenpulver der Haut an, der Finger wird aber nicht nass, was alle Teilnehmenden gern ausprobierten.

Nach etwa zwei Stunden schloss sich die Runde und unsere Gäste wurden mit folgendem Gedicht von Heinz EhrHARDT verabschiedet:

\section{Der Schauspieler}

Er sprach zu der Theaterleitung, nachdem er dreimal ausgespuckt: "Mein Name steht in dieser Zeitung nie eingerahmt, nie fettgedruckt!

Dabei spiel ich die längsten Rollen, mal bin ich heldisch, mal geduckt, ich probe auch, solang Sie wollen, doch niemals bin ich fettgedruckt!«

Ganz ohne Probe selbstverständlich starb gestern er, hat kaum gezuckt... Heut steht er in der Zeitung endlich schön eingerahmt und fettgedruckt!

Abb. 6: Cashewkerne sind sehr fetthaltig. Cashew-Bäume sind mit Mango verwandt. Mangobutter stammt aus den Steinkernen der Mangofrucht. 